\title{
Myosin regulatory light chain phosphorylation inhibits shortening velocities of skeletal muscle fibers in the presence of the myosin inhibitor blebbistatin
}

\author{
Melanie Stewart $\cdot$ Kathy Franks-Skiba • \\ Roger Cooke
}

Received: 12 August 2008/ Accepted: 7 November 2008/ Published online: 6 January 2009

(C) The Author(s) 2009. This article is published with open access at Springerlink.com

\begin{abstract}
Phosphorylation of skeletal myosin regulatory light chain (RLC) occurs in fatigue and may play a role in the inhibition of shortening velocities observed in vivo. Forces and shortening velocities were measured in permeabilized rabbit psoas fibers with either phosphorylated or dephosphorylated RLCs and in the presence or absence of the myosin inhibitor blebbistatin. Addition of $20 \mu \mathrm{M}$ blebbistatin decreased tensions by $\sim 80 \%$ in fibers, independent of phosphorylation. In blebbistatin maximal shortening velocities $\left(V_{\max }\right)$ at $30^{\circ} \mathrm{C}$, were decreased by $45 \%$ (3.2 \pm 0.34 vs. $5.8 \pm 0.18$ lengths/s) in phosphorylated fibers but were not inhibited in dephosphorylated fibers $(6.0 \pm 0.30$ vs. $5.4 \pm 0.30)$. In the presence of $20 \mu \mathrm{M}$ blebbistatin, $K_{\mathrm{m}}$ for $V_{\max }$ as a function of [ATP] was lower for phosphorylated fibers than for dephosphorylated fibers $(50 \pm 20$ vs. $330 \pm 84 \mu \mathrm{M})$ indicating that the apparent binding of ATP is stronger in these fibers. Phosphorylation of RLC in situ during fiber preparation or by addition of myosin light chain kinase yielded similar data. RLC phosphorylation inhibited velocity in blebbistatin at both 30 and $10^{\circ} \mathrm{C}$, unlike previous reports where RLC phosphorylation only affected shortening velocities at higher temperatures.
\end{abstract}

Keywords Myosin phosphorylation - Skeletal muscle · Blebbistatin · Motor protein

This work was supported by National Heart, Ling and Blood Institute Grant HL-32145.

M. Stewart $(\bowtie) \cdot$ K. Franks-Skiba $\cdot$ R. Cooke

Department of Biochemistry and Biophysics and The

Cardiovascular Research Institute, University of California San

Francisco, MC 2240, 600 16th Street, Genentech Hall, San

Francisco, CA 94158-2517, USA

e-mail: melanie.stewart@ucsf.edu

\section{Introduction}

Muscle fatigue is characterized by decreases in a number of muscle parameters including force and shortening velocity. Numerous studies have investigated the mechanism of inhibition but the precise mechanism is unknown (Fitts 1994; Westerblad and Allen 2003; Cooke 2007). Fatigue affects every system involved in muscle mechanics. It is known that the inhibition in function involves, in part, mechanisms that directly affect the interaction of actin and myosin, and these include product inhibition due to the accumulation of metabolites. Although replication of these metabolite levels in vitro results in a decrease in shortening velocities, the observed inhibition does not account for the extent seen in vivo (Fitts 1994; Westerblad et al. 1997, 2002; Myburgh 2004; Cooke 2007).

The role of phosphorylation in skeletal muscle is not as clear as it is in smooth muscle and is the subject of research by a number of groups. In smooth muscle it is well documented that the phosphorylation of the myosin regulatory light chain (RLC) is important in the control of activation of smooth muscle (Butler and Siegman 1998; Stull et al. 1990). In skeletal muscle RLC is phosphorylated by a dedicated calcium-calmodulin kinase, myosin light chain kinase (MLCK) (Pires et al. 1974) and dephosphorylated by a phosphatase (Morgan et al. 1976). Calcium activation of the kinase during heavy use and fatigue, leads to increased RLC phosphorylation, which provides a likely mechanism for the regulation of muscle mechanics under these conditions. A correlation between RLC phosphorylation and the force achieved in a twitch following a lengthy tetanus or a train of twitches first suggested a role for phosphorylation in twitch potentiation. (For review see (Sweeney et al. 1993). More conclusive evidence, that RLC phosphorylation was related to increased twitch tension, was found, in vitro, by 
Persechini and Cooke (Persechini et al. 1985), where it was shown that RLC phosphorylation increased tension in partially activated permeablized fibers. More definitively, twitch potentiation was absent, or greatly attenuated, in transgenic mice lacking MLCK (Zhi et al. 2005).

The effect of myosin RLC phosphorylation on shortening velocity has been studied extensively, is an active site of investigation and still the subject of debate. Crow and Kushmerick initially suggested that shortening velocities were inhibited by myosin RLC phosphorylation, from a correlation observed in mouse skeletal muscle during a sustained tetanus (Crow and Kushmerick 1982a, b). However, this hypothesis was disputed by Barsotti and Butler (Butler et al. 1983; Barsotti and Butler 1984) who found a dissociation between shortening velocities or ATPase activities and myosin RLC phosphorylation. No effect of myosin phosphorylation on tension or velocity was found in fully activated permeabilized fibers (Persechini et al. 1985; Sweeney and Kushmerick 1985; Sweeney and Stull 1990).

A number of small molecules are known to inhibit muscle tension, including phosphate, phosphate analogs, butane dione monoxime and blebbistatin. In the great majority of conditions where tension is inhibited, velocity is also inhibited (Dantzig and Goldman 1985; Chase et al. 1993; Seow et al. 1997; Regnier et al. 1999; Franks-Skiba et al. 2007). The inhibition of velocity has been hypothesized to be the result of an internal load imposed by nonworking myosin heads. The limited conditions in which velocity is not inhibited include vanadate (Pate et al. 1994), but only at high temperatures and in dephosphorylated fibers, and phosphate under most conditions. The present paper adds blebbistatin to the list of myosin inhibitors that inhibit tension but do not inhibit velocity, and shows that this property is controlled by phosphorylation.

Recent work from our laboratory, has found that under conditions where fiber tensions are partially inhibited, RLC phosphorylation inhibits the inhibition of shortening velocities, (Franks-Skiba et al. 2007; Karatzaferi et al. 2007). Analogs of $\mathrm{Pi}$ and the transition state with ADP.P., such as $\mathrm{BeF}_{3}, \mathrm{AlF}_{4}$ and $\mathrm{VO}_{4}$, bind to myosin at the nucleotide binding site, but populate different pre-power stroke states, and are thought to form structures similar to myosin in complex with ATP or ADP.Pi (Fisher et al. 1995; Smith and Rayment 1996). Addition of these analogs inhibits fiber tension but inhibits the maximum extrapolated velocity $\left(V_{\max }\right) V_{\max }$ differently. In the presence of vanadate, for example, RLC phosphorylation inhibits shortening velocity compared with fibers with dephosphorylated RLC; although isometric tensions were not affected (Franks-Skiba et al. 2007). A similar effect was found at low $\mathrm{pH}$ (6.2) and at high concentration of phosphate $(30 \mathrm{mM})$, (such found in fatigue in vivo), where RLC phosphorylation inhibited $V_{\max }$ relative to dephosphorylated RLC in fibers (Karatzaferi et al. 2007). However, little or no effect of phosphorylation on shortening velocity, was observed for the analogs $\mathrm{BeF}_{3}$, or $\mathrm{AlF}_{4}$ at pH 7 (Franks-Skiba et al. 2007). In the above studies, the effect of RLC phosphorylation on shortening velocity was found only at elevated temperatures, i.e. $30^{\circ} \mathrm{C}$ but not $10^{\circ} \mathrm{C}$. At the lower temperature most agents that inhibited tension, e.g. phosphate analogs or lower $\mathrm{pH}$, also inhibited shortening velocity and phosphorylation of the fibers had little or no effect.

Blebbistatin is a novel compound and a useful pharmacological tool for investigations of the molecular mechanisms of the actomyosin cycle. Blebbistatin was recently identified as a specific inhibitor of non-muscle myosin II, (Straight et al. 2003) which inhibited the contraction of the cleavage furrow without disrupting mitosis or the contractile ring assembly (Limouze et al. 2004). It has been subsequently shown to be an uncompetitive inhibitor that binds to a hydrophobic pocket at the apex of the $50 \mathrm{kDa}$ cleft, close to the $\gamma$-phosphate binding pocket (Allingham et al. 2005). Blebbistatin preferentially binds to the closed state (based on the position of switch II) in which ATP is hydrolyzed and results in the state Myosin॰ADP•P $\mathrm{P}_{\mathrm{i}}$ (Kovacs et al. 2004). Binding to the actin binding cleft would prevent this cleft from closing. We have used blebbistatin because it induces a known structure in myosin, with kinetics that have been thoroughly analyzed and it stabilizes the structure of the thick filament (Xu et al. 2008); a structure known to be disordered by myosin phosphorylation (Levine et al. 1996). In addition Blebbistatin does not bind to the nucleotide site, as do the phosphate analogs used in previous studies. Currently, a number of research groups are utilizing blebbistatin because of its specificity for myosin and the fact that it has little effect on the rest of the contractile apparatus.

Reported here are measurements of certain parameters of the mechanics of permeabilized rabbit psoas muscle fibers with phosphorylated or dephosphorylated myosin in the presence or absence of blebbistatin at different concentrations of ATP and different temperatures. Myosin phosphorylation inhibits velocity in the presence of blebbistatin, and lowers the $K_{\mathrm{m}}$ for $V_{\max }$ at a range of ATP concentrations. For the first time an effect of phosphorylation has been found at lower temperatures. This last observation has been attributed to the ability of blebbistatin to stabilize the binding of myosin heads to the core of the thick filament.

\section{Materials and methods}

Chemicals were purchased from Sigma (USA) Chemical Co, USA, except $( \pm)$ blebbistatin [( \pm -phenyl-1, 2, 3, 
4-tetrahydro-4-hydroxypyrrolo [2, 3-B]-7-methylquinolin4-one] or $(+)$ blebbistatin which were obtained from Toronto Research Chemicals, Inc., 2 Brisbane Road, North York, ON M3J 2J8 Canada. The racemic mixture was used as reports with regard to activity of each enantiomer have been mixed. Blebbistatin was dissolved in dimethylformamide (DMF) and stored at $-20^{\circ} \mathrm{C}$ before diluting with buffer shortly before use. Care was taken to limit its exposure to light as there is evidence that, exposed to certain wavelengths in the visible, it loses its effectiveness. Buffers were prepared as follows: relaxing buffer$120 \mathrm{mM} \mathrm{K}$-acetate, $5 \mathrm{mM} \mathrm{MgCl}{ }_{2}, 1 \mathrm{mM}$ EGTA, $5 \mathrm{mM} \mathrm{K}$ phosphate, $50 \mathrm{mM} 3$-(N-morpholino) propanesulfonic acid (MOPS) and $4 \mathrm{mM}$ ATP, unless otherwise stated, at $\mathrm{pH}$ 7.0 , ionic strength $=\sim 170 \mathrm{mM}$. Activating buffer was similarly prepared but with the addition of $1 \mathrm{mg} / \mathrm{ml}$ creatine kinase, $20 \mathrm{mM}$ creatine phosphate and $\mathrm{CaCl}_{2}$ added to pCa of 4.3 , ionic strength $\sim 190 \mathrm{mM}$. Lysis buffer: $26 \%$ glycerol, pH 4-6 ampholines, 30\% Triton X 100, 8 M urea, $92 \mathrm{mM}$ dithiothreitol (DTT). Pro-Q for staining isoelectric focusing gel was obtained from Molecular Probes, Eugene, OR.

\section{Preparation of muscle fibers}

Rabbits were sacrificed according to the protocols approved by the Institutional Animal Care and Use Committee. Muscle fibers were permeabilized by the method described in (Karatzaferi et al. 2007). Bundles of fibers were tied to supports, placed in glycerol solution $(120 \mathrm{mM}$ K-acetate, $5 \mathrm{mM} \mathrm{MgCl}_{2}, 5 \mathrm{mM}$ EGTA, $50 \mathrm{mM}$ MOPS set at $\mathrm{pH} 7.0$ and $50 \%$ glycerol $(\mathrm{v} / \mathrm{v}))$ and gently mixed overnight at $4^{\circ} \mathrm{C}$. Bundles were then placed in fresh solu-

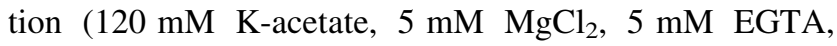
$50 \mathrm{mM}$ MOPS set at $\mathrm{pH} 7.0$ and $50 \%$ glycerol $(\mathrm{v} / \mathrm{v})) \mathrm{pH}$ 7.0 incubated at $4{ }^{\circ} \mathrm{C}$ for $4 \mathrm{~h}$ before transferring to $-20^{\circ} \mathrm{C}$ for storage. Permeabilized muscle fibers can be stored for up to several months without loss of integrity.

\section{Myosin phosphorylation. Preparation of fibers}

In most experiments phosphorylated myosin was prepared by the following method. Permeabilised rabbit psoas muscle fibers were incubated in glycerol solution as above that also contained phosphatase inhibitors and ATP (i.e. $5 \mathrm{mM}$ ATP, $20 \mathrm{mM} \mathrm{NaF}$ and $20 \mathrm{mM} \mathrm{K}$-phosphate, $\mathrm{pH}$ 7.0). The time course was approximately a week at $4^{\circ} \mathrm{C}$ with mixing.

In other experiments individual fibers with dephosphorylated myosin RLC were mounted onto the tensiometer and partially purified exogenous MLCK (see "Preparation of muscle fiber myosin light chain kinase" below) with calmodulin were added, and shortening velocities, tensions, etc. recorded. Specifically muscle fibers were incubated with relaxed rigor buffer with $\sim 300 \mu \mathrm{g} / \mathrm{ml}$ MLCK, $5 \mu \mathrm{g} /$ $\mathrm{ml}$ calmodulin, $5 \mathrm{mM} \mathrm{Pi}, 2 \mathrm{mM} \mathrm{CaCl}_{2}, 4 \mathrm{mM}$ ATP $\gamma \mathrm{S}$ for $30 \mathrm{~min}$ at $22^{\circ} \mathrm{C}$. Fibers were transferred to activating solution set at $5^{\circ} \mathrm{C}$ before measurement of shortening velocities at either 30 or $10^{\circ} \mathrm{C}$ as described below in "Measurement of the mechanics of muscle contraction".

Myosin phosphorylation. Isolectric focusing gel

Phosphorylation of muscle fiber RLC was determined using isoelectric focusing gels ( $\mathrm{pH} 4.0-6.0)$ as described previously (Franks-Skiba et al. 2007). Gels were stained with Pro-Q, which stains more strongly (approximately 4 times per $\mu \mathrm{g}$ of light chain loaded) phosphorylated serines and threonines; it also stains dephosphorylated light chains of myosin, but to a much lesser extent (see Fig. 1).

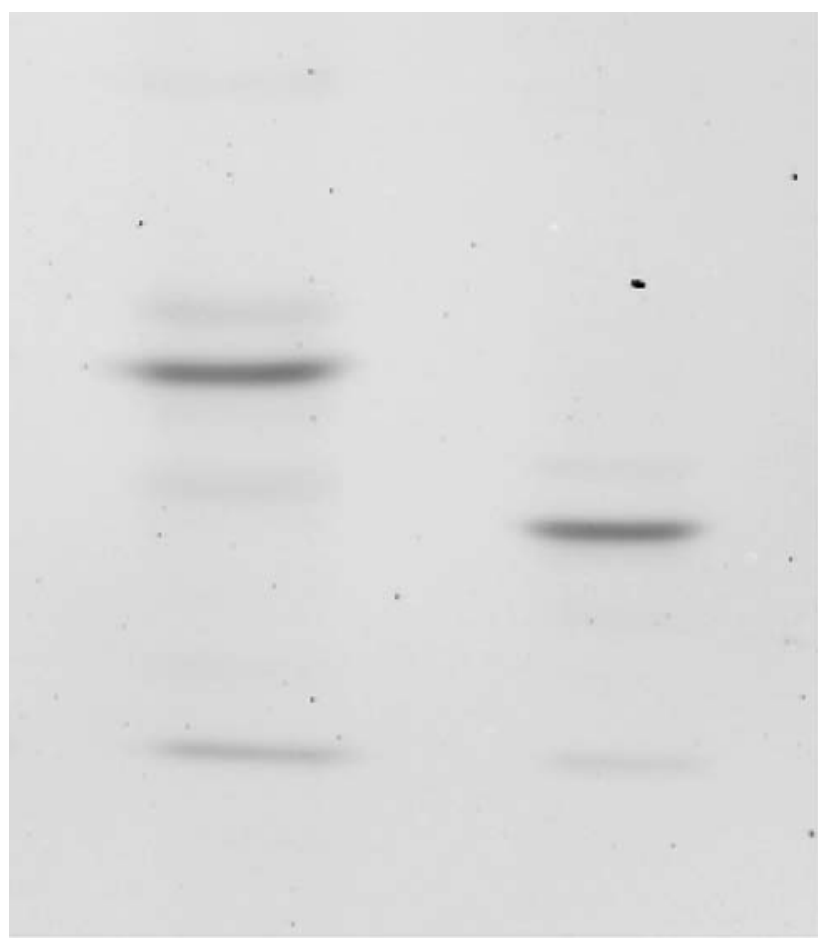

Fig. 1 Isoelectric focusing gel of two fiber preparations. Muscle fibers were phosphorylated in situ during preparation as described in Materials and Methods. Light chains were separated by an isoelectric gel, $\mathrm{pH} 4-6$, and stained with Pro-Q. Pro-Q has greater specificity for phosphorylated serines and threonines but also stains the dephosphorylated RLC. Pro-Q stains phosphorylated RLC 4 times more strongly than dephosphorylated RLC. The uppermost band is dephosphorylated RLC the second band is phosphorylated RLC and the lowest band is light chain 3. Lane 1 shows fibers where RLC is almost entirely dephosphorylated and lane 2 shows fibers where RLC is almost entirely phosphorylated. The amount of protein loaded in lane 1 is greater than that in lane 2 in order to observe the dephosphorylated band 
Preparation of myosin regulatory light chain kinase

Freshly excised muscle was washed in ice cold 2mMEDTA, extracted, minced, placed ice cold solution of $0.1 \mathrm{M} \mathrm{KH}_{2} \mathrm{PO}_{4}, 0.05 \mathrm{M} \mathrm{K}_{2} \mathrm{HPO}_{4}, 0.3 \mathrm{M} \mathrm{KCl}$, set at $\mathrm{pH} 6.5$ with stirring for $15 \mathrm{~min}$, centrifuged $(10,000 \mathrm{~g}, 10 \mathrm{~min}$, $4^{\circ} \mathrm{C}$ ) and the precipitate resuspended and centrifuged again as before. Precipitate was dissolved in a solution of $0.5 \mathrm{M}$ $\mathrm{KCl}, 20 \mathrm{mM} \mathrm{K}$-phosphate, $12.5 \mathrm{mM} \mathrm{MgCl}_{2}$ and $0.1 \mathrm{M}$ $\mathrm{CaCl}_{2}, \mathrm{pH} 8.0$ at room temperature. ATP was added to a final concentration of $5 \mathrm{mM}$ and the sample allowed to phosphorylate at room temperature for $30 \mathrm{~min}$. Following a series of washes MLCK is separated from myosin by centrifugation. This preparation follows protocols provided by Danuta Szczesna-Cordary, personal communication.

Measurement of the mechanics of muscle contraction

Mechanical measurements were obtained using the temperature-jump method (Karatzaferi et al. 2007). Single fibers, $\sim 0.5 \mathrm{~cm}$ in length by $\sim 60-70 \mu \mathrm{m}$ in diameter, or pairs, were mounted and fixed with vinyl adhesive between a solid state force transducer (Akers 801; Aksjelskapet Microelectronic, Hovtea, Norway) and an arm connected to a rapid motor (General Scanning, Inc., Watertown, MA, USA). The motor arm is adjusted to the point where the muscle fiber(s) are just no longer slack and then stretched $10 \%$. Sarcomere lengths were periodically measured with a laser at wavelength $638.2 \mathrm{~nm}$, and remained within acceptably intact limits of $\sim 2.2-2.4$. Typically fibers were first placed in cold $\left(5^{\circ} \mathrm{C}\right)$ relaxing buffer, before being transferred to cold $\left(5^{\circ} \mathrm{C}\right)$ activating buffer. Following a 2 min equilibration period fibers were periodically and rapidly transferred to and from a third solution containing activating buffer set at a temperature of 30 or $10^{\circ} \mathrm{C}$ according to the experiment and load clamps recorded. It has been found that measurements made during brief periods at the elevated temperature allows for a greater number of measurements to be made before fiber tension deteriorates beyond $10 \%$ of the original and some can be attributed to sarcomere heterogeneity. Transfers to $30^{\circ} \mathrm{C}$ solution and measurements recorded were $\sim 1 \mathrm{~s}$. Blebbistatin is light sensitive and precautions were taken to ensure that solutions containing blebbistatin had minimum exposure to light (Sakamoto et al. 2005). Shortening velocities were measured as described previously (Karatzaferi et al. 2007).

Force-velocity data from isotonic releases were analyzed by a least squares, nonlinear fit of the data to the Hill equation given below

$V=V_{\max } \cdot\left(\alpha / P_{0}\right)\left(1-P / P_{0}\right) /\left(\alpha / P_{0}+P / P_{0}\right)$ where $\mathrm{P}$ is tension at velocity $V, P_{0}$ is isometric tension, $V_{\text {max }}$ is the maximum velocity extrapolated to zero tension and $\alpha / P_{0}$ is a variable related to the curvature of the relationship, the smaller this variable the more curved is the relationship. The fit, was performed using Kaleidagraph 3.6, which provides $95 \%$ confidence limits for the variables, which are given in the text. Force velocity curves were composed of 20-40 data points that were obtained from 5 to 10 different fibers.

\section{Results}

Force inhibition with blebbistatin

Blebbistatin decreases isometric tension in fibers to the same extent irrespective of the phosphorylation status of myosin, Fig. 2. Control fibers were incubated in medium containing equivalent volumes of DMF and showed no such decrease in tension. Figure 2 also shows that the concentration of blebbistatin necessary to produce a decrease of $80 \%$ in tension is $\sim 15 \mu \mathrm{M}$. Subsequent experiments were conducted at $20 \mu \mathrm{M}$ blebbistatin to ensure that at least $75 \%$ inhibition of force was maintained.

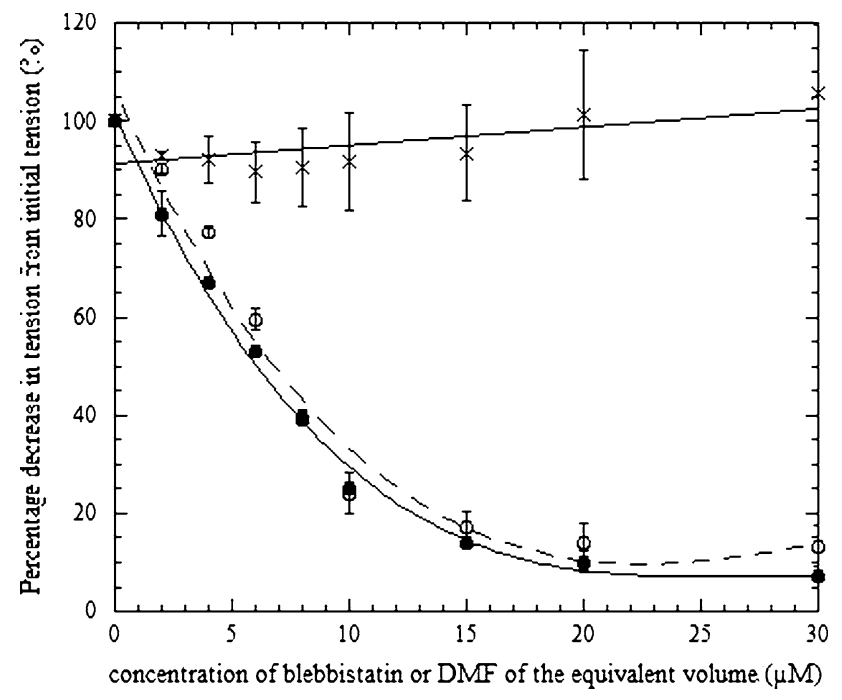

Fig. 2 Tension is shown as a function of the concentration of blebbistatin. Individual muscle fibers were mounted on to the tensiometer and placed in relaxing buffer plus the specified concentrations of blebbistatin and set at $5^{\circ} \mathrm{C}$. Control fibers were incubated in the concentration of DMF equivalent to that used in the experiments with blebbistatin. Following activation fibers were then transferred to activating solution with/without blebbistatin or DMF set at $30^{\circ} \mathrm{C}$ for $\sim 2 \mathrm{~s}$ and tensions recorded. Empty circles represent data for fibers with dephosphorylated RLC in the presence of blebbistatin, filled circles that for phosphorylated RLC in the presence of blebbistatin and X's represent fibers with phosphorylated RLC incubated in solutions with a volume of DMF added that was equivalent to the corresponding blebbistatin concentration. Data represent means \pm SEMs for at least 3 fibers 
Effect of myosin phosphorylation and the myosin inhibitor blebbistatin on shortening velocities at $30^{\circ} \mathrm{C}$

Fiber shortening velocities were measured in the presence and absence of $20 \mu \mathrm{M}$ blebbistatin. In the presence of $20 \mu \mathrm{M}$ blebbistatin, maximal shortening velocities for fibers with phosphorylated RLC, $3.2 \pm 0.34$, were decreased by $47 \%$ compared with fibers with dephosphorylated RLC, $6.0 \pm 0.30$, see Figs. 3 and 4. The inhibition of shortening velocity by RLC phosphorylation is only seen in the presence of blebbistatin; in its absence phosphorylation had no effect on shortening velocity, see Fig. 5a, b. Blebbistatin has no effect on shortening velocities in fibers with dephosphorylated RLC even though forces were decreased by at least $75 \%$. Shortening velocity inhibition by blebbistatin is only seen in phosphorylated fibers, shown in Fig. 5a. There was no systematic variation in the curvature correlated with myosin phosphorylation, in agreement with previous observations (Sweeney and Kushmerick 1985; Franks-Skiba et al. 2007; Karatzaferi et al. 2007). Similar differences in shortening velocities were also found using incubations that contained $10 \mu \mathrm{M}$ blebbistatin.

Effect of the myosin inhibitor blebbistatin and myosin phosphorylation on shortening velocities in permeabilized rabbit psoas muscle fibers at $10^{\circ} \mathrm{C}$

Our previous work had found that the inhibition of fiber shortening velocity by phosphorylation occurred only at higher temperatures and not at $10^{\circ} \mathrm{C}$, a temperature where many studies are carried out due to greater fiber stability. Thus we repeated our studies of blebbistatin at the lower temperature. The data obtained at $10^{\circ} \mathrm{C}$ was qualitatively similar to that obtained at $30^{\circ} \mathrm{C}$, see Fig. 6. Addition of $20 \mu \mathrm{M}$ blebbistatin inhibited tension equally in phosphorylated and dephosphorylated fibers and to the same extent as it did at $30^{\circ} \mathrm{C}$. In the absence of blebbistatin, phosphorylation had no effect on either $V_{\max }$ or $\alpha / P_{0}$, as observed previously under other conditions (Sweeney and Kushmerick 1985; Franks-Skiba et al. 2007; Karatzaferi et al. 2007). The addition of $20 \mu \mathrm{M}$ blebbistatin did not affect the force velocity curve in dephosphorylated fibers but inhibited maximal shortening velocity for fibers with phosphorylated RLC by $57 \%$, Fig. 6 . This inhibition is a little greater than that seen at $30^{\circ} \mathrm{C}, 47 \%$.

Effect of different isomers of blebbistatin

Reports of the stereospecificity of blebbistatin have been mixed. (+) Blebbistatin has been found to be inactive for ATPase in human platelet non muscle myosin II (Straight et al. 2003) whereas Kovacs et al. (Kovacs et al. 2004)
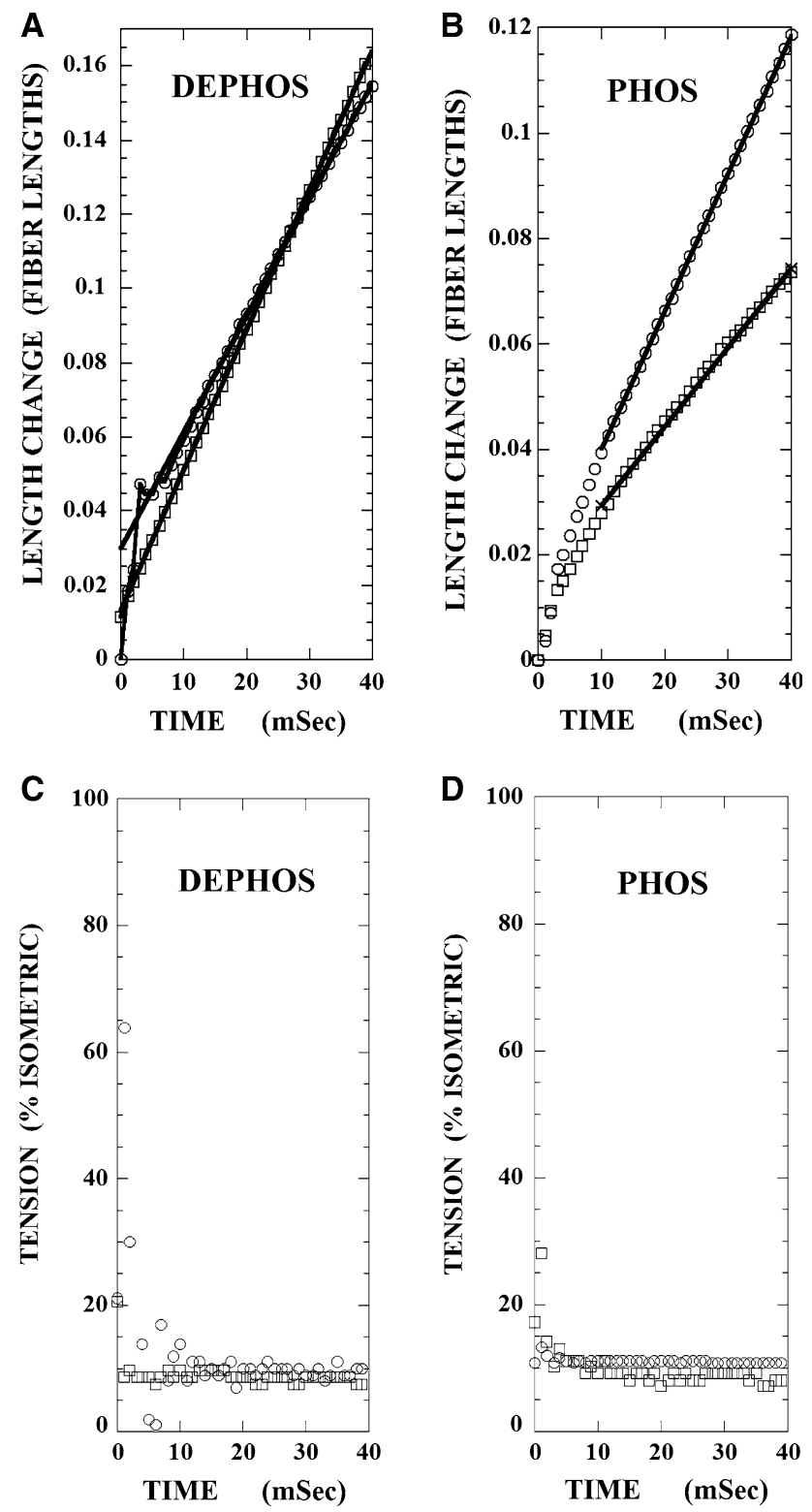

Fig. 3 Isotonic release data for rabbit psoas muscle fibers under the four conditions studied. $\mathbf{a}$ and $\mathbf{b}$ show the position of the motor arm to which the fibers were attached as a function of time during the clamp. c and $\mathbf{d}$ show release tensions as a function of time following initiation of a tension clamp to $\sim 10 \%$ of isometric tension. Open circles, data obtained in the absence of blebbistatin, open squares, data obtained in the presence of blebbistatin. The slope of a least-squares fit to the position versus time data over the period $10-40 \mathrm{~ms}$ following release is also shown (solid lines) and was taken as the shortening velocity. Note that in the absence of blebbistatin there is a greater elastic component early in the position trace due to the greater tensions generated. Dephosphorylated fibers plus blebbistatin isotonic tension $=12 \%$ velocity $=3.15$ lengths $/ \mathrm{s}$; in the absence of blebbistatin isotonic tension $=11 \%$ velocity $=3.36$. Phosphorylated fibers plus blebbistatin isotonic tension $=10 \%$ velocity $=1.97$ lengths $/ \mathrm{s}$; in the absence of blebbistatin isotonic tension $=11 \%$ velocity $=2.8$ lengths $/ \mathrm{s}$

have found similar data for both enantiomers in rabbit skeletal muscle myosin. The effect of $(+)$ blebbistatin on tensions was determined. Pairs of fibers were mounted on 


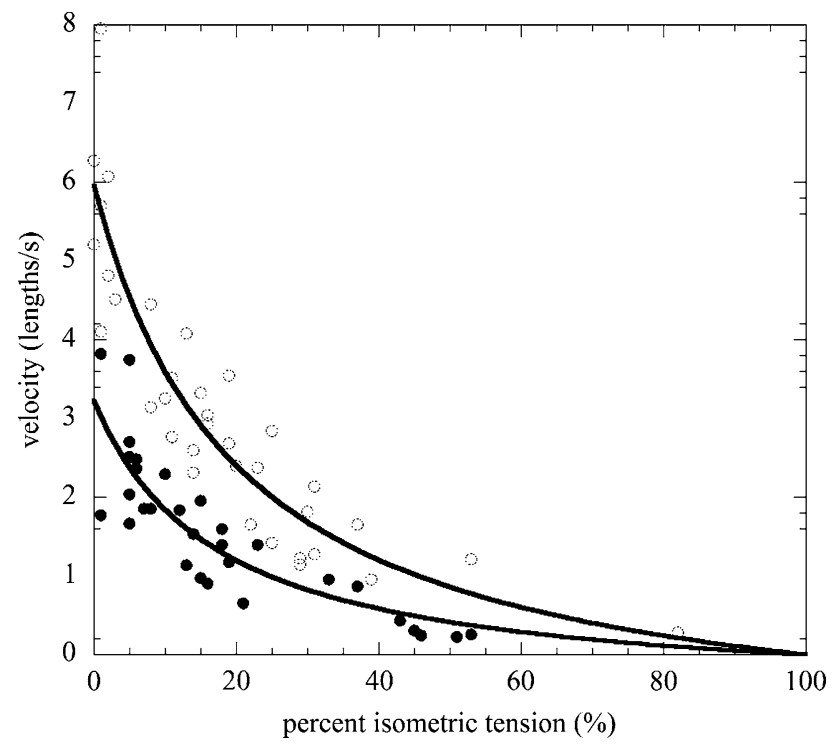

Fig. 4 The effect of myosin phosphorylation on shortening velocities of muscle fibers in the presence of $20 \mu \mathrm{M}$ blebbistatin at $30^{\circ} \mathrm{C}$. Filled circles represent data from fibers with phosphorylated RLC, $V_{\max }$ $3.2 \pm 0.34, \alpha /$ Po $0.2 \pm 0.06$; empty circles represent that from fibers with dephosphorylated RLC, $V_{\max } 6.0 \pm 0.30, \alpha / \mathrm{Po} 0.2 \pm 0.04$. In this and following figures the lines show fits to the data using the Hill equation. The fit, was performed using Kaleidagraph 3.6, which provides $95 \%$ confidence limits for the variables. Force velocity curves were composed of 20-40 data points that were obtained from 5 to 10 different fibers
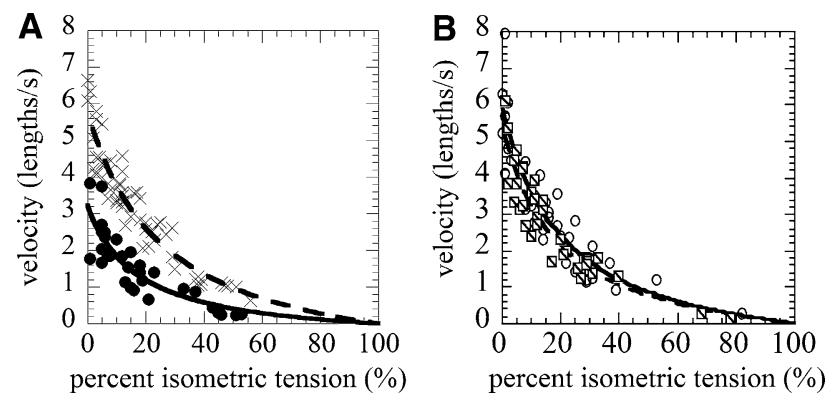

Fig. 5 The effect of $20 \mu \mathrm{M}$ blebbistatin on shortening velocities of muscle fibers at $30^{\circ} \mathrm{C}$ with phosphorylated RLC (a) or dephosphorylated RLC (b). a Filled circles and solid line represent data from fibers with phosphorylated RLC incubated in the presence of $20 \mu \mathrm{M}$ blebbistatin; $V_{\max } 3.2 \pm 0.34, \alpha / \mathrm{Po} 0.17 \pm 0.06$; X's and dashed line represent that for phosphorylated RLC in the absence of blebbistatin; $V_{\max } 5.8 \pm 0.18, \alpha /$ Po $0.26 \pm 0.03$. b Empty circles and solid line represent dephosphorylated fibers in the presence of $20 \mu \mathrm{M}$ blebbistatin; $V_{\max } 6.0 \pm 0.30 \alpha / \mathrm{Po} 0.2 \pm 0.03$; and crossed boxes and dashed line show data in the absence of blebbistatin; $V_{\max } 5.4 \pm 0.3$, $\alpha /$ Po $0.2 \pm 0.03$. Data were fit using the Hill equation, solid lines, dephosphorylated RLC, dashed lines phosphorylated RLC

the tensiometer and placed in relaxing buffer set at $5^{\circ} \mathrm{C}$, then transferred to active solution at $5^{\circ} \mathrm{C}$ for $2 \mathrm{~min}$, lastly moved to activating solution set at $30^{\circ} \mathrm{C}$ for $\sim 2 \mathrm{~s}$, forces measured and the fiber returned relaxing buffer. This procedure was repeated with the same fiber placed in $20 \mu \mathrm{M}$

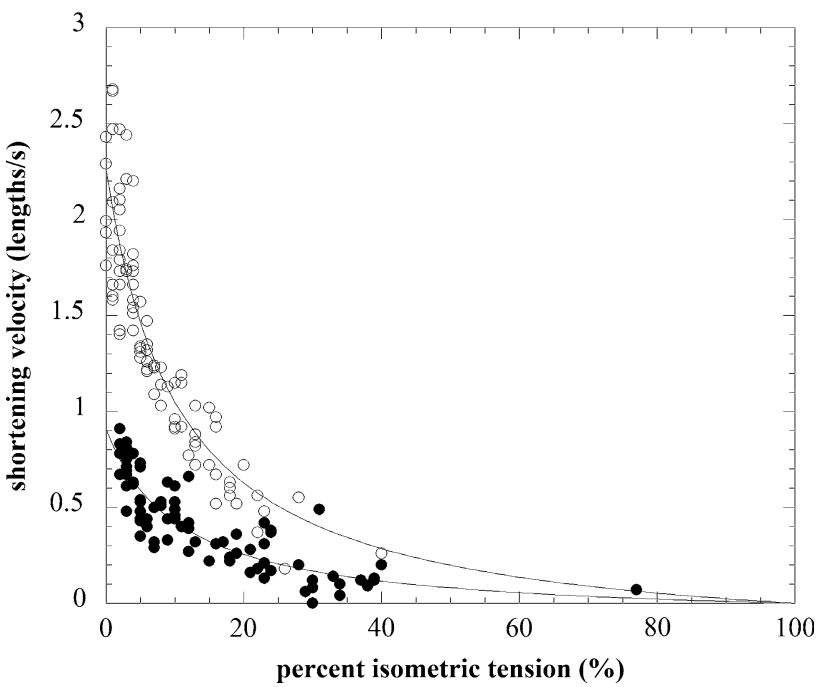

Fig. 6 The effect of myosin phosphorylation on shortening velocities in the presence of $20 \mu \mathrm{M}$ blebbistatin at $10^{\circ} \mathrm{C}$. This is the same experiment as shown in Fig. 4 but with a lower temperature. Filled circles represent data for fibers with phosphorylated RLC; $V_{\max }$ $0.9 \pm 0.06, \alpha / \mathrm{Po} 0.11 \pm 0.02$; empty circles that for dephosphorylated RLC, $V_{\max } 2.3 \pm 0.2, \alpha / \mathrm{Po} 0.07 \pm 0.02$. In the absence of blebbistatin fiber shortening velocities were not affected by phosphorylation: phosphorylated RLC, $V_{\max } 2.12 \pm 0.04 \alpha /$ Po $0.10 \pm 0.01$; dephosphorylated RLC, $V_{\max } 2.26 \pm 0.07, \alpha / \mathrm{Po} 0.11 \pm 0.01$. Data were fit using the Hill equation

(+) blebbistatin. On average a $29 \%$ decrease in tension was found which does not compare with a $80 \%$ decrease found with $20 \mu \mathrm{M}( \pm)$ blebbistatin. Therefore, we find that the $(+)$ isomer has partial activity.

\section{Measurement of $K_{\mathrm{m}}$ [ATP]}

Previous work has shown that in the presence of vanadate, RLC phosphorylation also lowers the $K_{\mathrm{m}}$ for $V_{\max }$ as a function of ATP. The maximum velocity of shortening is shown as a function of the ATP concentration for fibers inhibited by $20 \mu \mathrm{M}$ blebbistatin, Fig. 7. In the presence of blebbistatin the fibers have a greater apparent affinity for ATP, shown by the lower value of $K_{\mathrm{m}}$ in phosphorylated fibers, $K_{\mathrm{m}}=50 \pm 20 \mu \mathrm{M}$, than in dephosphorylated fibers, $K_{\mathrm{m}}=330 \pm 84 \mu \mathrm{M}$. This effect is similar to that seen in vanadate (Franks-Skiba et al. 2007). In the absence of inhibitors phosphorylation has no effect on $K_{\mathrm{m}}$ (FranksSkiba et al. 2007).

Effect of phosphorylation of individual fibers using purified MLCK

In our previous work we had achieved phosphorylated fibers by in situ phosphorylation during the period of storage in the rigor/glycerol solution. In the presence of ATP and the phosphatase inhibitors, phosphate and KF, 


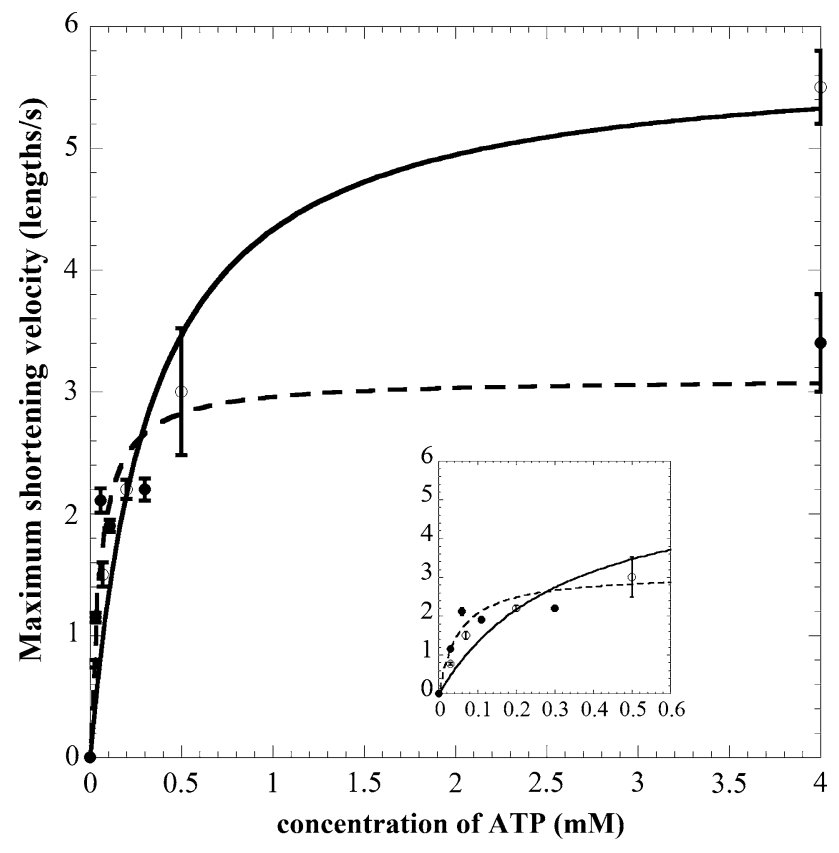

Fig. 7 The effect of myosin phosphorylation on maximal shortening velocity as a function of ATP concentration in the presence of $20 \mu \mathrm{M}$ blebbistatin at $30^{\circ} \mathrm{C}$ : fibers with phosphorylated RLC, filled circles and fibers with dephosphorylated RLC, empty circles. Error bars on the points represent the standard error from 3 to 4 different force velocity curves. The maximum shortening velocity was determined by fitting force-velocity data using the Hill plot and extrapolated maximal shortening velocities were plotted here against [ATP]. A fit to the data define $K_{\mathrm{m}}=300 \mu \mathrm{M} \pm 84 \mu \mathrm{M}, \quad V_{\max }=5.7 \pm 0.5$ lengths/s for dephosphorylated fibers compared with $K_{\mathrm{m}}=50 \mu \mathrm{M} \pm 20 \mu \mathrm{M}, V_{\max }=3.1 \pm 0.3$ lengths/s, for phosphorylated fibers. Inset shows more detail in the range of [ATP] $0-0.6 \mu \mathrm{M}$. Errors on the parameters represent $95 \%$ confidence limits determined by the fit to the data

fibers became phosphorylated over about a week, while in the absence of these reagents they became dephosphorylated. As this procedure may have changed other properties of the fibers, it was important to show that the effects of RLC phosphorylation could be reproduced by direct phosphorylation of the RLC using MLCK.

Fibers were mounted on the tensiometer and incubated in a partially purified MCLK, calmodulin and ATP $\gamma \mathrm{S}$. The ATP analog, ATP $\gamma \mathrm{S}$, was used because it is a good substrate for MLCK but a poor substrate for activating tension in the fibers. In addition the thiophosphate attached to serine on the RLC is resistant to the phosphatase. Individual muscle fibers were mounted on the tensiometer and phosphorylated by addition of MLCK and calmodulin at $22^{\circ} \mathrm{C}$ for $30 \mathrm{~min}$, as described in "Materials and methods". This temperature was chosen as higher temperatures resulted in destabilization of the fiber sarcomere lattice structure. Following incubation, shortening velocities were recorded at $30^{\circ} \mathrm{C}$, using the temperature jumps as described above. The results presented in Table 1 show that RLC phosphorylated using this procedure produced the same
Table 1 Force-velocity parameters of fibers phosphorylated by added myosin light chain kinase

\begin{tabular}{llllr}
\hline Incubation $^{\mathrm{a}}$ & Blebbistatin $^{\mathrm{b}}$ & $V_{\max }^{\mathrm{c}}$ & $\alpha / P_{\mathrm{o}}$ & $\%^{\mathrm{d}}$ \\
\hline- MLCK & 0 & $5.3 \pm 0.2$ & $0.18 \pm 0.02$ & 0 \\
- MLCK & $20 \mu \mathrm{M}$ & $4.9 \pm 0.4$ & $0.26 \pm 0.02$ & -8 \\
+ MLCK & 0 & $4.6 \pm 0.2$ & $0.17 \pm 0.02$ & -14 \\
+ MLCK & $20 \mu \mathrm{M}$ & $3.5 \pm 0.2$ & $0.27 \pm 0.06$ & -35 \\
\hline
\end{tabular}

${ }^{a}$ Fibers were incubated in buffer containing $4 \mathrm{mMATP} \gamma \mathrm{S}$, MLCK, calmodulin and $20 \mu \mathrm{Mblebbistatin,} \mathrm{in} \mathrm{rigor} \mathrm{buffer} \mathrm{at} 22^{\circ} \mathrm{C}$, for $30 \mathrm{~min}$, see "Materials and methods" for details. The fiber was then transferred to activating solution for measurement of forces and shortening velocities

${ }^{b}$ Concentration of blebbistatin in measurement of force-velocity

${ }^{c}$ Following incubation, shortening velocities were measured at $30^{\circ} \mathrm{C}$ and data fitted to the Hill equation (Hill 1938), data represent 95\% confidence limits on the fit to the data

${ }^{\mathrm{d}}$ Percentage change from control, i.e. row 1 fibers with dephosphorylated myosin incubated without MLCK, calmodulin or blebbistatin

inhibition of shortening velocity in the presence of blebbistatin as found for the fibers phosphorylated during storage in glycerol. Figure 8 shows that the level of phosphorylation achieved using this procedure was high.

\section{Discussion}

\section{Relation to previous work}

This work is an extension of our efforts to understand the mechanism of the inhibition of shortening velocity during muscle fatigue, and in particular the role of myosin phosphorylation in this process. Our previous work has found that, in conditions where sizable populations of myosin heads in states not generating force, myosin phosphorylation inhibits velocity. These conditions have included the addition of vanadate, which is known to trap myosin in a pre-power stroke state, and a combination of higher phosphate $(30 \mathrm{mM})$ and lower $\mathrm{pH}$ (6.2). However, not all phosphate analogs produce this effect, for example $\mathrm{AlF}_{4}$ or $\mathrm{BeF}_{3}$ at $\mathrm{pH} 7.0$ have no such effect and it was not observed previously except at high temperatures, $30^{\circ} \mathrm{C}$. We find no effect of myosin phosphorylation on either velocity or tension in the absence of inhibitors, in fibers in which all myosin heads are involved in active cycles with actin.

The mechanism of phosphorylation induced inhibition of velocity

The mechanism by which phosphorylation induces inhibition of shortening velocity can be explained by its effect on the array of myosin heads bound to the thick filament. 


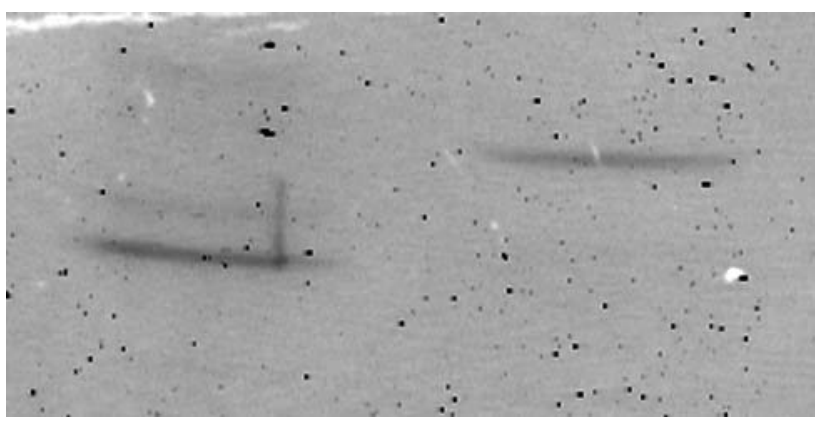

Fig. 8 Isoelectric focusing gel of muscle fibers following incubation with or without $4 \mathrm{mM} \mathrm{ATP} \gamma \mathrm{S}$, exogenous MLCK and calmodulin in the presence of $20 \mu \mathrm{M}$ blebbistatin, at $22^{\circ} \mathrm{C}, 30 \mathrm{~min}$. Each lane represents protein from a single muscle fiber. The left lane is the result of the above incubation and shows that the myosin was indeed phosphorylated. The right lane is from a fiber incubated in precisely the same manner but without MLCK and calmodulin and shows no evidence of myosin phosphorylation

When myosin heads bind to the thick filament they form an ordered array. It is the disruption of this order that is believed to be the mechanism of inhibition of shortening velocity. The array itself was first visualized in resting frog muscles by electron microscopy and X-ray diffraction (reviewed in (Huxley and Faruqi 1983) and has since been shown in a variety of muscle types (Levine 1993). The array is labile, and an example of disruption to the array can be found in mammalian muscles, where order is only seen at higher temperatures (Wray 1987; Xu et al. 2003). In normal physiological conditions such as that in relaxed fibers, and probably also in fibers with large populations of non-force generating heads, these heads can be sequestered by binding to the thick filament where they are sterically unable to reach and interact with actin filaments. This effect will only operate in fibers with dephosphorylated myosin. In contrast phosphorylation of myosin causes disruption of the ordered array, increasing the fraction of myosin heads in a disordered configuration in the space around the thick filament, see Fig. 9 (Levine et al. 1996), which means that these heads can more readily bind with actin in non-force generating states, State 3 in Fig. 9.

We have previously observed the close correlation induced between the release of myosin heads from the thick filament array and the inhibition of velocity. In normal conditions all myosin heads are undergoing active cycles, and few heads, if any, are bound to the core of the thick filament. Here myosin phosphorylation has no apparent effect on velocity. Under conditions where the cycle is inhibited by a reagent(s), we find that the velocity is inhibited along with the tension under conditions where the myosin heads are not bound to the core of the thick filament. These conditions include lower temperatures, or phosphorylated fibers, both of which disrupt the array. If the array is stable then velocity does not tend to be

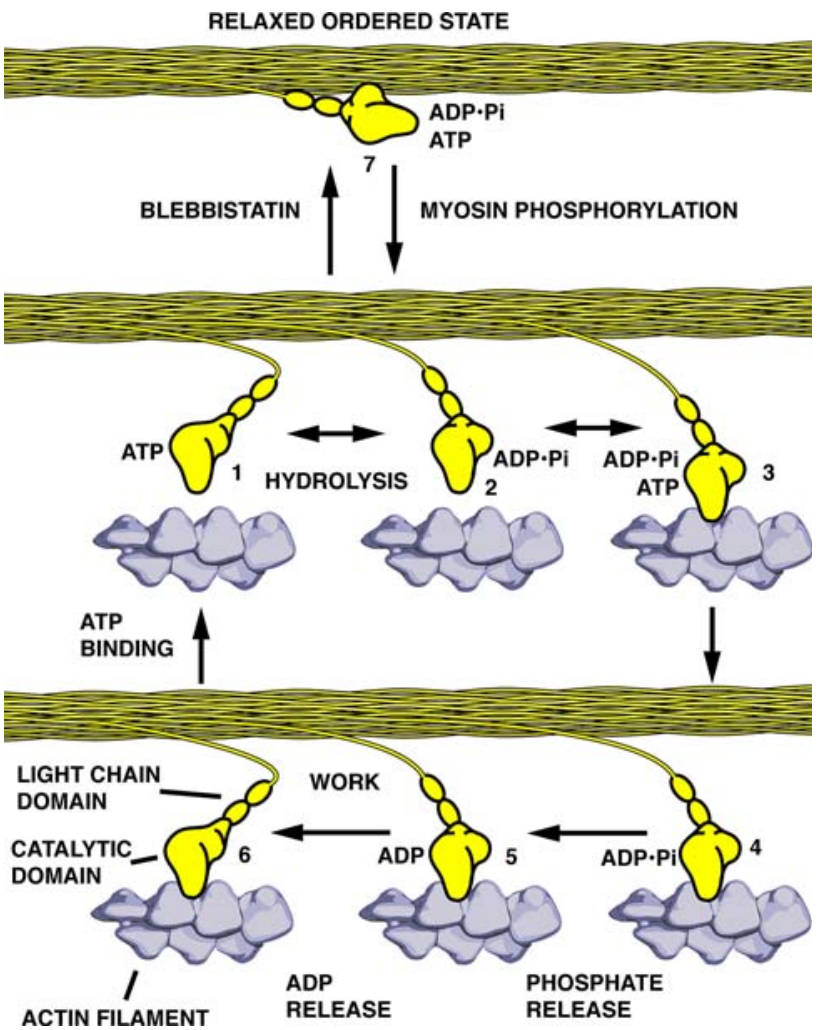

Fig. 9 The actomyosin cycle. State 1 represents myosin dissociated from actin following the binding of ATP. In state 2 ATP is hydrolyzed which alters the myosin structure to the pre-power stroke conformation. In state 3 myosin binds weakly to actin and does not generate force. Following isomerization to produce state 4, force is generated. In state 5 phosphate is released, actin forms a stronger bond with myosin and force is produced. Release of ADP brings the cycle to state 6 where the rigor bond is formed - the tight binding of actin and myosin. State 7 shows a myosin head bound to the thick filament. The presence of blebbistatin stabilizes this ordered state (Xu et al. 2008); whereas myosin phosphorylation disorders it (Levine et al. 1996)

inhibited as for dephosphorylated fibers in vanadate. Vanadate stabilizes the array to a greater extent than $\mathrm{BeF}_{3}$ (Wray 1987; Xu et al. 2003), and shortening velocities of dephosphorylated fibers are inhibited in $\mathrm{BeF}_{3}$, but not in vanadate. At lower temperatures, the array is unstable, and all of the phosphate analogs inhibit both tension and velocity. We also observed that in dephosphorylated fibers inhibited by vanadate, there was an abrupt change in velocity, which was inhibited below $22^{\circ} \mathrm{C}$ but not above (Pate et al. 1994). Such an abrupt change suggests involvement of a highly cooperative structure, and the thick filament provides a structure capable of such a degree of cooperativity.

A major difference between the new observations and the previous ones is that dephosphorylated fibers inhibited by blebbistatin have fast shortening velocities, equal to control fibers, and that myosin phosphorylation has the same ability to inhibit velocity at $10^{\circ} \mathrm{C}$ as it does at $30^{\circ} \mathrm{C}$. 
The observations that dephosphorylated fibers inhibited at $10^{\circ} \mathrm{C}$ by blebbistatin, are fast, but that phosphorylated fibers are slow, strongly support the stability of the ordered thick filament array as playing a role in the inhibition of velocity. Thus the inhibition of velocity by phosphorylation can be connected to disruption of this array, which is its only established structural effect.

The hypothesis discussed above suggests that velocity is inhibited when there are large populations of myosin heads in non force states that are capable of interacting with the thin filament. The inhibition of velocity depends on the properties of the non-cycling heads. Why would the presence of these non-force generating myosin heads interacting with the thin filament inhibit velocity? One hypothesis is that they bind transiently to the filament and exert a drag. Inhibition of sliding filament has been found with such binding (Cuda et al. 1997).

However, the observations of changes in $K_{\mathrm{m}}$ complicate the above explanation. The value of $K_{\mathrm{m}}$ is not affected by phosphorylation, in fully activated fibers in the absence of some inhibitory agent such as blebbistatin, or vanadate. In the presence of two of these inhibitors, vanadate and now blebbistatin, phosphorylated fibers have a much lower value of $K_{\mathrm{m}}$, indicating apparent stronger binding of ATP, and possibly other nucleotides as well. Thus the effect of phosphorylation on $K_{\mathrm{m}}$ requires the presence of myosin heads, presumably bound weakly to actin in non-force states. Such heads could be on the same myosin molecule, or on adjacent ones. Other possibilities include direct interaction of the regulatory light chain with the active site, affecting binding of nucleotides. Communication between the active site and a phosphorylated serine on the regulatory light chain has been observed previously (Mazhari et al. 2004). However this mechanism does not explain the lack of an effect of phosphorylation on fiber velocity in the absence of a large population of non-force cross-bridges. A preliminary report, which described the inhibition of velocity by myosin phosphorylation using an in vitro assay, in which actin filaments move over a surface coated with myosin, also suggests that there is some direct effect of myosin phosphorylation on the binding of nucleotides (Greenberg et al. 2008). There was no thick filament structure in these assays, however there may have been a population of myosin heads that could bind weakly to actin but were sterically unable to execute power strokes.

Each of the two hypotheses discussed above have data that supports them. At this point there are no data that rule out either hypothesis. It is also possible that the velocities are influenced by some completely different mechanism involving non-crossbridge elements such as titin or Cprotein, however there are no data that support such mechanisms.
Properties of the myosin-blebbistatin complex

Blebbistatin binds to a cleft, which begins at the nucleotide binding site and ends at the actin binding site. The cleft divides the molecule into two regions known as the upper $50 \mathrm{kDa}$ and lower $50 \mathrm{kDa}$ domains (Allingham et al. 2005). This cleft must close before myosin can form a strong bond with actin and blebbistatin is thought to bind in such a way as to prevent closure. Blebbistatin binds to the myosin.ADP.Pi complex with high affinity and interferes with phosphate release; the step that is generally accepted as involved in the transition to the force production steps. Blebbistatin arrests the myosin cycle in states that are either detached from actin, or bind only weakly to it. (Kovacs et al. 2004). Thus with blebbistatin we are investigating the mechanical behavior of a myosin state that is well defined structurally. The state that is populated in the presence of blebbistatin is an analog of a state that is also highly populated during fatigue.

Of particular importance for our studies is the observation that Blebbistatin stabilizes the binding of myosin heads to the core of the thick filament ( $\mathrm{Xu}$ et al. 2008). Thus our observation that shortening velocities are not inhibited by blebbistatin in dephosphorylated fibers, but inhibited in phosphorylated fibers, at both 10 and $30^{\circ} \mathrm{C}$, provides additional evidence that the array is involved in the mechanism of inhibition as discussed above.

Partial inhibition of fibers by reagents such as vanadate or blebbistatin results in two populations of myosin heads. Because the binding and release of the inhibitors is slow compared to the cycle time, a fiber whose tension has been inhibited by $80 \%$, has $\sim 20 \%$ of its heads undergoing active cycles, and $80 \%$ in non-force states, with slow exchange between the two populations. Because myosin phosphorylation does not appear to inhibit the cycle of the normally cycling cross bridges in the uninhibited fibers, the different effects of phosphorylation seen with different inhibitors must be due to the interactions between the cycling heads and the non-cycling heads, as only the noncycling heads are different.

\section{Summary}

The inhibition of shortening velocities in fatigue in skeletal muscle has not been completely explained by known mechanisms. Alterations in concentrations of metabolites account for some but not all of this inhibition and evidence from this laboratory and others has clearly demonstrated that myosin phosphorylation plays an important role.

The recently discovered myosin inhibitor blebbistatin, like that of other such inhibitors, e.g. vanadate, have revealed important information regarding the role of myosin phosphorylation in the inhibition of shortening 
velocities in skeletal muscle. The mechanism of action for blebbistatin in myosin, is now well established and its specificity makes it a unique and a very effective phar-

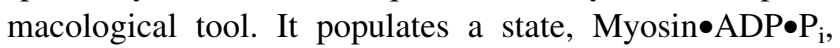
which mimics a state populated during fatigue. In conditions where this non-force generating state is populated, myosin phosphorylation significantly inhibits shortening velocities. In the absence of an elevated population of nonforce states, myosin phosphorylation has no effect on velocity. Thus our results provide strong support for the hypothesis that myosin phosphorylation is involved in the inhibition of shortening velocities during fatigue in vivo.

One mechanism proposed to explain the results presented here is that phosphorylation of myosin prevents the binding of myosin heads to the core of the thick filament, forming an ordered array (Levine et al. 1996). The inability of myosin heads to lie in this configuration means that heads are more able to interact with actin thus generating a population of heads in non-force generating states. Conversely dephosphorylated myosin heads are sequestered and are sterically unable to reach and interact with actin filaments. The data, however, do not rule out the possibility that myosin phosphorylation inhibits velocity by directly affecting the binding of nucleotides.

Acknowlegdments We thank Dr. Tom Purcell for assistance with preparation of figures for this manuscript.

Open Access This article is distributed under the terms of the Creative Commons Attribution Noncommercial License which permits any noncommercial use, distribution, and reproduction in any medium, provided the original author(s) and source are credited.

\section{References}

Allingham JS, Smith R, Rayment I (2005) The structural basis of blebbistatin inhibition and specificity for myosin II. Nat Struct Mol Biol 12:378-379

Barsotti RJ, Butler TM (1984) Chemical energy usage and myosin light chain phosphorylation in mammalian skeletal muscle. J Muscle Res Cell Motil 5:45-64

Butler TM, Siegman MJ (1998) Control of cross-bridge cycling by myosin light chain phosphorylation in mammalian smooth muscle. Acta Physiol Scand 164:389-400

Butler TM, Siegman MJ, Mooers SU, Barsotti RJ (1983) Myosin light chain phosphorylation does not modulate cross-bridge cycling rate in mouse skeletal muscle. Science 220:1167-1169

Chase PB, Martyn DA, Kushmerick MJ, Gordon AM (1993) Effects of inorganic phosphate analogs on stiffness and unloaded shortening of skinned fibres from rabbit. J Physiol 460:231-246

Cooke R (2007) Modulation of the actomyosin interaction during fatigue of skeletal muscle. Muscle Nerve 36:756-777

Crow MT, Kushmerick MJ (1982a) Correlated reduction of velocity of shortening and the rate of energy utilization in mouse fasttwitch muscle during a continuous tetanus. J Gen Physiol 82:703-722

Crow MT, Kushmerick MJ (1982b) Myosin light chain phosphorylation is associated with a decrease in the energy cost for contraction in fast twitch mouse muscle. J Biol Chem 257:21212124

Cuda G, Pate E, Cooke R, Sellers JR (1997) In vitro actin filament sliding velocities produced by mixtures of different types of myosin. Biophys J 72:1767-1779

Dantzig JA, Goldman YE (1985) Suppression of muscle contraction by vanadate. Mechanical and ligand binding studies on glycerolextracted rabbit fibers. J Gen Physiol 86:305-327

Fisher AJ, Smith CA, Thoden JB, Smith R, Sutoh K, Holden HM, Rayment I (1995) X-ray structures of the myosin motor domain of dictyostelium discoideum complexed with Mgadp-Center-DotBefx And Mgadp-Center-Dot-Alf4. Biochemistry 34:8960-8972

Fitts RH (1994) Cellular mechanisms of muscle fatigue. Physiol Rev 74:49-94

Franks-Skiba K, Lardelli R, Goh G, Cooke R (2007) Myosin light chain phosphorylation inhibits muscle fiber shortening velocity in the presence of vanadate. Am J Physiol Regul Integr Comp Physiol 292:R1603-R1612

Greenberg M, Pant K, Watt J, Mealy T, Jones M, Szczesna-Cordary D, Moore J (2008) The effects of myosin regulatory light chain phosphorylation on striated muscle myosin motility and force production. Biophys J 94:2280

Huxley HE, Faruqi AR (1983) Time-resolved X-ray diffraction studies on vertebrate striated muscle. Annu Rev Biophys Bioeng $12: 381-417$

Karatzaferi C, Franks-Skiba K, Cooke R (2007) Inhibition of shortening velocity of skinned skeletal muscle fibers in conditions that mimic fatigue. Am J Physiol Regul Integr Comp Physiol 294:R948-R955

Kovacs M, Toth J, Hetenyi C, Malnasi-Csizmadia A, Sellers JR (2004) Mechanism of blebbistatin inhibition of myosin II. J Biol Chem 279:35557-35563

Levine RJ (1993) Evidence for overlapping myosin heads on relaxed thick filaments of fish, frog, and scallop striated muscles. J Struct Biol 110:99-110

Levine RJ, Kensler RW, Yang Z, Stull JT, Sweeney HL (1996) Myosin light chain phosphorylation affects the structure of rabbit skeletal muscle thick filaments. Biophys J 71:898-907

Limouze J, Straight AF, Mitchison T, Sellers JR (2004) Specificity of blebbistatin, an inhibitor of myosin II. J Muscle Res Cell Motil 25(4-5):337-341

Mazhari SM, Selser CT, Cremo CR (2004) Novel sensors of the regulatory switch on the regulatory light chain of smooth muscle Myosin. J Biol Chem 279:39905-39914

Morgan M, Perry SV, Ottaway J (1976) Myosin light-chain phosphatase. Biochem J 157:687-697

Myburgh KH (2004) Can any metabolites partially alleviate fatigue manifestations at the cross-bridge? Med Sci Sports Exerc 36: 20-27

Pate E, Wilson G, Bhimani M, Cooke R (1994) Temperature dependence of the inhibitory effects of orthovanadate on shortening velocity in fast skeletal muscle. Biophys J 66:1554-1562

Persechini A, Stull JT, Cooke R (1985) The effect of myosin phosphorylation on the contractile properties of skinned rabbit skeletal muscle fibers. J Biol Chem 260:7951-7954

Pires E, Perry SV, Thomas MA (1974) Myosin light-chain kinase, a new enzyme from striated muscle. FEBS Lett 41:292-296

Regnier M, Chase PB, Martyn DA (1999) Contractile properties of rabbit psoas muscle fibres inhibited by beryllium fluoride. $\mathrm{J}$ Muscle Res Cell Motil 20:425-432

Sakamoto T, Limouze J, Combs CA, Straight AF, Sellers JR (2005) Blebbistatin, a myosin II inhibitor, is photoinactivated by blue light. Biochemistry 44:584-588

Seow CY, Shroff SG, Ford LE (1997) Detachment of low-force bridges contributes to the rapid tension transients of skinned rabbit skeletal muscle fibres. J Physiol 501(Pt 1):149-164 
Smith CA, Rayment I (1996) X-ray structure of the magnesium (II) • ADP - vanadate complex of the Dictyostelium discoideum myosin motor domain to $1.9 \AA$ resolution. Biochemistry 35:5404-5417

Straight AF, Cheung A, Limouze J, Chen I, Westwood NJ, Sellers JR, Mitchison TJ (2003) Dissecting temporal and spatial control of cytokinesis with a myosin II Inhibitor. Science 299:1743-1747

Stull JT, Bowman BF, Gallagher PJ, Herring BP, Hsu LC, Kamm KE, Kubota Y, Leachman SA, Sweeney HL, Tansey MG (1990) Myosin phosphorylation in smooth and skeletal muscles: regulation and function. Prog Clin Biol Res 327:107-126

Sweeney HL, Bowman BF, Stull JT (1993) Myosin light chain phosphorylation in vertebrate striated muscle: regulation and function. Am J Physiol 264:C1085-C1095

Sweeney HL, Kushmerick MJ (1985) Myosin phosphorylation in permeabilized rabbit psoas fibers. Am J Physiol 249:C362-C365

Sweeney HL, Stull JT (1990) Alteration of cross-bridge kinetics by myosin light chain phosphorylation in rabbit skeletal muscle: implications for regulation of actin-myosin interaction. Proc Natl Acad Sci USA 87:414-418
Westerblad H, Allen DG (2003) Cellular mechanisms of skeletal muscle fatigue. Adv Exp Med Biol 538:563-570

Westerblad H, Allen DG, Lannergren J (2002) Muscle fatigue: lactic acid or inorganic phosphate the major cause? News Physiol Sci $17: 17-21$

Westerblad H, Bruton JD, Lannergren J (1997) The effect of intracellular $\mathrm{pH}$ on contractile function of intact, single fibres of mouse muscle declines with increasing temperature. J Physiol 500(Pt 1):193-204

Wray J (1987) Temperature dependence of the relaxed pattern from rabbit psoas fibers. J Muscle Res Cell Motil 8:62a

Xu S, Offer G, Gu J, White HD, Yu LC (2003) Temperature and ligand dependence of conformation and helical order in myosin filaments. Biochemistry 42:390-401

Xu S, White HD, Offer G, Yu L (2008) Stabilisation of the helical order of myosin filaments by blebbistatin. Biophys J 94:629

Zhi G, Ryder JW, Huang J, Ding P, Chen Y, Zhao Y, Kamm KE, Stull JT (2005) Myosin light chain kinase and myosin phosphorylation effect frequency-dependent potentiation of skeletal muscle contraction. Proc Natl Acad Sci USA 102:17519-17524 